\title{
Uso de Ontologias para Agentes Conversacionais no Contexto de Ensino-Aprendizagem: Uma Revisão Sistemática da Literatura
}

\author{
Cleon X. Pereira Júnior ${ }^{1,5}$, Rodrigo E. Francisco ${ }^{2,5}$, Luciane de F. Silva ${ }^{3,5}$, \\ Ernesto F. Veiga ${ }^{4}$, Fabiano A. Dorça ${ }^{5}$, Márcia A. Fernandes ${ }^{5}$ \\ ${ }^{1}$ Instituto Federal Goiano (IF Goiano) \\ Campos Belos - GO - Brasil \\ ${ }^{2}$ Instituto Federal Goiano (IF Goiano) \\ Morrinhos - GO - Brasil \\ ${ }^{3}$ Instituto de Informática - Centro Universitário do Triângulo (UNITRI) \\ Uberlândia - MG - Brasil \\ ${ }^{4}$ Instituto de Informática - Universidade Federal de Goiás (UFG) \\ Goiânia - GO - Brasil \\ ${ }^{5}$ Faculdade de Computação - Universidade Federal de Uberlândia (UFU) \\ Uberlândia - MG - Brasil \\ \{cleon.junior, rodrigo.francisco\} @ifgoiano.edu.br
\{lucianefatsilva, ernestofonsecaveiga\} @gmail.com \\ \{fabianodor, marcia\} @ufu.br
}

\begin{abstract}
This paper presents a Systematic Review of Literature (RSL) in order to verify the contribution of Ontologies to Conversational Agents $(C A)$ in the teaching-learning context. The results indicate that the use of Ontologies in CAs is promising, since they allow actions in different domains, with different methods of interaction, inclusion of aspects of affectivity, and the need to meet non functional requirements such as usability and performance. Despite the benefits found, it is known that the creation of ontologies is costly. However, it is necessary to continue the research in a practical and experimental approach to seek the limits of the performance of such systems in real situations.
\end{abstract}

Resumo. Este artigo apresenta uma Revisão Sistemática da Literatura (RSL) com o objetivo de verificar a contribuição das Ontologias para os Agentes Conversacionais $(A C)$ no contexto de ensino-aprendizagem. Os resultados apontam que o uso das Ontologias nos ACs é promissor, pois possibilitam atuações em diferentes domínios, com diferentes métodos de interação, inclusão de aspectos de afetividade, e a necessidade de atender a requisitos não funcionais como usabilidade e performance. Apesar dos benefícios encontrados, sabe-se que é oneroso a criação das ontologias. Entretanto, destaca-se a necessidade da continuidade da pesquisa em uma abordagem prática e experimental visando buscar os limites da atuação de tais sistemas em situações reais. 
VI Congresso Brasileiro de Informática na Educação (CBIE 2017)

Anais do XXVIII Simpósio Brasileiro de Informática na Educação (SBIE 2017)

\section{Introdução}

A tecnologia tem se mostrado cada vez mais presente em todas as modalidades de ensino. Com isso, alguns desafios se destacam, especialmente quando se refere às modalidades à distância ou semipresenciais, tais como os relacionados à melhor abordagem do conteúdo. A área de Inteligência Artificial (IA), por meio de sistemas adaptativos e tutores pedagógicos virtuais, executa um papel importante para a educação, como exemplo, na forma de apresentar o conteúdo para o estudante.

A literatura considera que o comportamento do estudante é relevante para o processo de aprendizagem, conforme já observado por teorias psicopedagógicas, como o modelo Ortony, Clore, Collins (OCC) [Ortony et al. 1990], o que se relaciona aos aspectos emocionais, que influenciam na predisposição de comportamentos positivos ou negativos. Teorias, como o modelo OCC, auxiliam na criação de agentes em ambientes virtuais e dão suporte aos mesmos, por meio de emoções, aumentando a interatividade do estudante com o sistema, corroborando com as teorias de Piaget e Vigotski quanto aos aspectos da socialização no de ensino-aprendizagem [Palangana 2015].

Num contexto colaborativo, os Ambientes Virtuais de Aprendizagem (AVA) podem encontrar problemas devido à ausência física dos participantes, o nível heterogêneo de integrantes do grupo, a necessidade de suporte instrucional, dentre outros. Tais problemas são considerados como fatores que contribuem para gerar desmotivação dos estudantes. Dentre as aplicações utilizadas para evitar tal desmotivação, destaca-se o uso de Agentes Conversacionais (AC).

Um Agente Conversacional (AC), também conhecido como chatterbot ou chatbot, é definido como uma ferramenta responsável por designar uma conversa inteligente (tanto em forma de fala quanto em escrita) com um ou mais humanos [Al-Zubaide and Issa 2011]. Desenvolvido com técnicas de IA, os ACs possuem aplicações em áreas como saúde (no auxílio de pacientes acerca do tratamento de doenças, por exemplo), comércio e educação.

No contexto educacional, em um AVA, estes podem gerar um diálogo com o aprendiz de forma natural, instigando-o, por exemplo, em conteúdos presentes em uma atividade. A literatura relata o uso de redes neurais [Serban et al. 2016], lógica fuzzy [Fabian et al. 2006], dentre outras técnicas de Inteligência Artificial para a construção de AC, e, além disso, aborda aspectos arquiteturais, como apresentado por [Moraes and Machado 2016] ao propor AC para o Enino, que permite gerar respostas automáticas ao estudantes, na intenção de recomendar materiais instrucionais. No entanto, há um desafio ao adaptá-los para atuarem em diferentes contextos. Este trabalho visa investigar o uso das ontologias como estratégia para resolver tal problema.

Uma ontologia, na perspectiva da Ciência da Computação, é uma descrição formal e explícita dos conceitos de um domínio, o que possibilita relacionar dados à semântica definida na ontologia. Assim, as ontologias podem contribuir no cenário de ensino-aprendizagem de diferentes maneiras, sendo uma delas o suporte ao desenvolvimento dos Agentes Conversacionais. Por exemplo, utilizar duas ontologias, sendo uma do domínio "senso comum" e outra do domínio "sentimentos" permite que o agente consiga planejar o diálogo que será executado com o aluno, abordando assuntos que contribuem para melhoria emocional do aprendiz e que esteja relacionado à sua cultura 
VI Congresso Brasileiro de Informática na Educação (CBIE 2017)

Anais do XXVIII Simpósio Brasileiro de Informática na Educação (SBIE 2017)

[Ong and Soriano 2014].

Nota-se que há contribuições de AC na aprendizagem colaborativa, porém os mesmos não estão limitados a este domínio. Neste sentido, o presente trabalho apresenta uma Revisão Sistemática da Literatura (RSL) que visa responder à questão de pesquisa: Como as ontologias podem contribuir para AC no contexto de ensino-aprendizagem?. A RSL também buscou investigar os requisitos funcionais e não funcionais de um AC para o contexto de ensino-aprendizagem e os benefícios e problemas do uso de ontologias para construir estes ACs.

Este artigo está organizado como se segue. A Seção 2 apresenta a metodologia aplicada na RSL, que esta foi dividida em planejamento, execução e resultados. A Seção 3 apresenta os resultados da RSL após os critérios de inclusão e exclusão. Por fim, na Seção 4 são apresentadas as conclusões obtidas por meio da RSL.

\section{Metodologia}

Esta RSL segue as instruções elaboradas por [Kitchenham 2004], cuja condução se subdivide em três etapas: Planejamento, Execução e Resultados. O objetivo desta revisão é verificar como estão os trabalhos que envolvem ACs e ontologias no contexto de ensinoaprendizagem. Assim, as etapas a seguir são importantes para delimitar tal contexto.

\subsection{Planejamento}

Para responder a questão principal desta pesquisa ( Como as ontologias podem contribuir para ACs no contexto de ensino-aprendizagem?) foram definidas outras quatro questões de pesquisa, sendo elas:

- QP1: Quais requisitos funcionais são identificados em ACs no contexto de ensinoaprendizagem?

- QP2: Quais requisitos não-funcionais são identificados em ACs no contexto de ensino-aprendizagem?

- QP3: Quais os benefícios na adoção de ontologias em ACs no contexto de ensinoaprendizagem?

- QP4: Quais as dificuldades na adoção de ontologias em ACs no contexto de ensino-aprendizagem?

Foi realizada uma busca nas bases de artigos científicos, considerando os trabalhos de 2011 a 2017 (Com a data limite de 01/04/2017) e selecionadas as bases de pesquisa: IEEEXplorer Digital Library, ACM Digital Library, Science Direct, Scopus e Springer. A escolha das bases ocorreu devido estas possuirem mecanismos de buscas online, utilizando filtros por ano e tipo de publicação, e, além disso, conterem trabalhos relacionados a temas de Computação. A Tabela 1 descreve as strings usadas nas buscas e a Tabela 2 apresenta os critérios de inclusão e exclusão, que foram construídos considerando as questões de pesquisa.

Tabela 1. Strings de busca para a seleção de artigos

\begin{tabular}{|c|c|}
\hline Idioma & \multicolumn{1}{|c|}{ String } \\
\hline Português & ("agente conversacional" or "chatbot*" or"chatterbot*") and ("ontolog*") and ("aprendizagem") \\
\hline Inglês & ("conversational agent" or "chatbot*" or "chatterbot*") and ("ontolog*") and ("learn") \\
\hline
\end{tabular}


VI Congresso Brasileiro de Informática na Educação (CBIE 2017)

Anais do XXVIII Simpósio Brasileiro de Informática na Educação (SBIE 2017)

Tabela 2. Critérios de Inclusão e Exclusão

\begin{tabular}{|l|l|l|}
\hline Critérios & ID & Descrição \\
\hline \multirow{4}{*}{ Exclusão } & E1 & Texto completo não disponível para acesso na Web \\
\cline { 2 - 3 } & E2 & Escrito em outro idioma \\
\cline { 2 - 3 } & E3 & Trabalho não aborda o uso de ontologias em chatbot no contexto de ensino-aprendizagem. \\
\cline { 2 - 3 } & E4 & Não é um capítulo de livro com resumo ou artigo de periódico ou de conferência \\
\cline { 2 - 3 } & E5 & Publicação duplicada \\
\hline \multirow{4}{*}{ Inclusão } & I1 & Aborda os requisitos funcionais de chatbots no contexto de ensino-aprendizagem. \\
\cline { 2 - 3 } & I2 & Aborda os requisitos não-funcionais de chatbots no contexto de ensino-aprendizagem. \\
\cline { 2 - 3 } & I3 & Aborda os benefícios da adoção de ontologias em chatbots no contexto de ensino-aprendizagem. \\
\cline { 2 - 3 } & I4 & Aborda as dificuldades da adoção de ontologias em chatbots no contexto de ensino-aprendizagem. \\
\hline
\end{tabular}

\subsection{Execução}

A etapa de execução (presente como segunda fase da RSL) é composta de três partes: identificação dos trabalhos, seleção e extração. A identificação dos trabalhos visa executar o plano da RSL e obter a lista de artigos. A seleção ocupa-se da leitura dos artigos realizada de uma maneira mais geral (título, palavras-chave e resumo). A extração exige que seja feita a leitura completa dos artigos, buscando realizar uma síntese com ênfase nas questões de pesquisa levantadas no planejamento. A Tabela 3 apresenta os números da etapa de execução desta pesquisa.

Tabela 3. Etapa de Execução

\begin{tabular}{|l|l|l|l|}
\hline Fonte & Execução & Seleção & Extração \\
\hline ACM & 1 & 0 & 0 \\
IEEE & 6 & 2 & 2 \\
Springer & 92 & 6 & 3 \\
Scopus & 13 & 6 & 5 \\
Science Direct & 1 & 0 & 0 \\
\hline Total & $\mathbf{1 1 3}$ & $\mathbf{1 4}$ & $\mathbf{1 0}$ \\
\hline
\end{tabular}

O processo de execução das Strings trouxeram 113 artigos. Destes 90 foram rejeitados por se enquadrarem em um dos 4 primeiros critérios de exclusão e 9 foram apontados como duplicados. Na etapa de seleção restaram somente 14 artigos. Após leitura na íntegra, 4 foram rejeitados pelo critério E3, restando os 10 trabalhos que serão discutidos. A Tabela 4 apresentam os trabalhos classificados pelos critérios de inclusão.

Tabela 4. Trabalhos incluídos após a etapa de execução

\begin{tabular}{|c|c|c|}
\hline Inclusão & Referência & Quantidade \\
\hline I1 & $\begin{array}{c}\text { [Abdelkefi and Kallel 2016], [Freitas et al. 2014], [Al-Zubaide and Issa 2011], } \\
\text { [Hussain et al. 2012], [Lundqvist et al. 2013], [Ong and Soriano 2014] }\end{array}$ & 6 \\
I2 & $\begin{array}{r}\text { [Fonte et al. 2012], [Abdelkefi and Kallel 2016], [Al-Zubaide and Issa 2011], } \\
\text { [Ong and Soriano 2014], [Hussain et al. 2012] }\end{array}$ & 4 \\
& $\begin{array}{r}\text { [Fonte et al. 2012], [Abdelkefi and Kallel 2016], [Freitas et al. 2014], } \\
\text { [Al-Zubaide and Issa 2011], [Hussain et al. 2012], [Lundqvist et al. 2013], } \\
\text { [Ong and Soriano 2014] }\end{array}$ & 8 \\
& $\begin{array}{r}\text { [Fonte et al. 2012], [Abdelkefi and Kallel 2016], [Freitas et al. 2014], } \\
\text { IAl-Zubaide and Issa 2011], [Lundqvist et al. 2013] }\end{array}$ & 4 \\
\hline
\end{tabular}


VI Congresso Brasileiro de Informática na Educação (CBIE 2017)

Anais do XXVIII Simpósio Brasileiro de Informática na Educação (SBIE 2017)

Foi observado que nos trabalhos apresentados, diferentes ontologias foram utilizadas na construção do AC. A Tabela 5 apresenta uma relação das ontologias especificadas. Alguns trabalhos não deixaram explícitos o nome da ontologia.

Tabela 5. Ontologias utilizadas em cada projeto

\begin{tabular}{|c|l|}
\hline Ontologia utilizada & Referências \\
\hline ConceptNet Adaptada, SenticNet & {$[$ Ong and Soriano 2014] } \\
\hline Cyc & [Augello et al. 2011] \\
\hline SEQUEEL Quality Ontology, Teaching Product Ontology & {$[$ Lundqvist et al. 2013] } \\
\hline OntBot & [Al-Zubaide and Issa 2011] \\
\hline HEO & {$[$ Hussain et al. 2012] } \\
\hline MKB & {$[$ Lundqvist et al. 2013] } \\
\hline DBpedia & [Serón and Bobed 2016] \\
\hline
\end{tabular}

\section{Resultados}

A leitura dos trabalhos considerando os critérios da RSL permitiu realizar uma síntese dos dados. Isso possibilitou responder a cada uma das quatro questões de pesquisa e formar uma visão geral sobre o assunto.

\section{QP1: Quais requisitos funcionais são identificados em chatbots no contexto de ensino-aprendizagem?}

Com as leituras dos trabalhos, foi possível levantar três principais requisitos funcionais, que é esperado em um AC, para que este seja utilizado no contexto de ensinoaprendizagem. A Tabela 6 apresenta os trabalhos classificados de acordo com os requisitos funcionais.

Tabela 6. Requisitos funcionais de um AC para o ensino-aprendizagem

\begin{tabular}{|l|l|}
\hline $\begin{array}{l}\text { Requisitos } \\
\text { Funcionais }\end{array}$ & Referências \\
\hline RF1 - Diferentes domínios & $\begin{array}{l}\text { [Al-Zubaide and Issa 2011], [Freitas et al. 2014], } \\
\text { [Serón and Bobed 2016], [Lundqvist et al. 2013] }\end{array}$ \\
\hline $\begin{array}{l}\text { RF2 - Diferentes Métodos de } \\
\text { Interação }\end{array}$ & $\begin{array}{l}\text { [Abdelkefi and Kallel 2016], [Hussain et al. 2012], } \\
\text { [Ong and Soriano 2014] }\end{array}$ \\
\hline RF3 - Afetividade & [Ong and Soriano 2014], [Hussain et al. 2012] \\
\hline
\end{tabular}

Espera-se de um AC que este possa reagir com diferentes tipos de diálogos, portanto o domínio do conhecimento é importante e não deve ser limitado, principalmente quando se trata de uma arquitetura mais genérica e que será aplicada a diferentes situações. [Al-Zubaide and Issa 2011] apresenta o OntBot, um chatbot que tenta agir como humano na compreensão sobre o que o usuário fala, usando informações sobre o escopo da pergunta. O OntBot se assemelha ao TempLang [Freitas et al. 2014] que consegue responder a perguntas que exigem definições ou explicações e não se limita a um único domínio. Outro exemplo é o VOX System [Serón and Bobed 2016], um agente conversacional corporificado que por meio de gestos e interações permite fazer uma filtragem e uma busca do domínio que compete o diálogo. [Lundqvist et al. 2013] desenvolveram um 
VI Congresso Brasileiro de Informática na Educação (CBIE 2017)

Anais do XXVIII Simpósio Brasileiro de Informática na Educação (SBIE 2017)

método para implementação de chatbots que podem mudar o conhecimento do domínio facilmente e expandir a base de conhecimento.

Por muitas vezes, quando se trata de ACs no âmbito de ensino-aprendizagem, é imaginável apenas um diálogo em forma de texto. Trabalhos recentes têm mostrado diferentes formas de interação entre o usuário e o AC. No caso de [Abdelkefi and Kallel 2016] é utilizado o texto escrito e a fala para que haja uma melhor interação. Já o VOX System, por ser um AC corporificado (interage com o ambiente por meio de um corpo virtual), recorre ao uso de meios verbais e até não verbais (como gestos) para gerar um diálogo mais próximo ao que ocorre em um ambiente real. Por último, o sistema IMACA (Intelligente Multimodal Affective Conversational Agent) utiliza video, audio e texto para interagir e incorporar o AC dos dados coletados dessas interações [Hussain et al. 2012].

Com relação a afetividade, duas pesquisas externam a importância de um AC ser dotado de afetividade para que haja maior interação com o usuário. O sistema IMACA coleta dados de diferentes fontes para conseguir criar um AC próximo ao esperado pelo usuário e também o tornar afetivo. Já o trabalho desenvolvido por [Ong and Soriano 2014] apresenta um AC para auxiliar na mudança de estados afetivos de estudantes. Portanto, o AC age em estudantes que apresentam desmotivação com diálogos motivadores por meio de ontologias de senso comum e de sentimentos.

Ainda sobre os requisitos funcionais, para uma aprendizagem móvel utilizando um AC, segundo [Abdelkefi and Kallel 2016], é necessário que o mesmo seja sensível ao contexto e tenha suporte a vários idiomas. Desta forma se torna acessível em qualquer lugar e possui a capacidade de tradução simultânea.

QP2: Quais requisitos não-funcionais são identificados em chatbots no contexto de ensino-aprendizagem?

Tabela 7. Requisitos Não Funcionais identificados

\begin{tabular}{|l|l|}
\hline $\begin{array}{l}\text { Requisitos } \\
\text { Não Funcionais }\end{array}$ & Referências \\
\hline RNF1 - Performance & $\begin{array}{c}\text { [Al-Zubaide and Issa 2011], [Abdelkefi and Kallel 2016], [Augello et al. 2011], } \\
\text { [Ong and Soriano 2014] }\end{array}$ \\
\hline RNF2 - Interoperabilidade & [Al-Zubaide and Issa 2011], [Augello et al. 2011], [Hussain et al. 2012] \\
\hline RNF3 - Segurança & [Abdelkefi and Kallel 2016] \\
\hline RNF4 - Usabilidade & [Abdelkefi and Kallel 2016], [Augello et al. 2011] \\
\hline
\end{tabular}

A Tabela 7 apresenta os requisitos não-funcionais identificados nos ACs para o contexto de ensino-aprendizagem. É notado o surgimento de interoperablidade (RNF2), requisito não tão comum em outros domínios, porém mais visivel quando se trata de aplicações com ontologias. Os artigos [Al-Zubaide and Issa 2011] e [Augello et al. 2011] reforçam esta ideia. O OntBot apresenta a construção de uma ontologia para ser aplicada em ACs e deixa explícito a interoperabilidade em seu projeto. [Augello et al. 2011] apresenta um framework e ressalta a capacidade de comunicar com outros sistemas para que o AC consiga alcançar o objetivo de uma conversa em linguagem natural. $O$ trabalho que apresenta o sistema IMACA argumenta sobre a necessidade de interoperabilidade, neste caso, vários subsistemas se comunicando para gerar um sistema completo.

$\mathrm{O}$ requisito não funcional mais esperado em um AC é a questão da Performance. Espera-se que os sistemas consigam gerar respostas de forma instantânea para os usuá- 
VI Congresso Brasileiro de Informática na Educação (CBIE 2017)

Anais do XXVIII Simpósio Brasileiro de Informática na Educação (SBIE 2017)

rios. Conforme a Tabela 7, é possível ver que quatro trabalhos deixam explícitos a necessidade de tratar a escalabilidade em seus sistemas para que estes consigam operar. A segurança é um requisito não funcional reforçado na construção do sistema MobiSpeech [Abdelkefi and Kallel 2016]. O módulo de segurança foi descrito utilizando a plataforma Aglet da IBM. A usabilidade também é discutida nos trabalhos por se tratar de interações entre máquinas e seres humanos.

\section{QP3: Quais os benefícios na adoção de ontologias em chatbots no contexto de ensino-aprendizagem?}

Todos os trabalhos que utilizam ontologias na construção de ACs contribuiram para a geração da base de conhecimento utilizada pelo AC. A extração automática de ontologias de corpos de texto [Morrison and Rus 2013], que pode ser feita via aprendizagem de máquina e mineração de dados, beneficia o uso de ontologias em chatbots, melhorando sua compreensão e a produtividade na construção da ontologia. Porém, alguns trabalhos destacam pontos específicos nesta construção. A seguir, de forma breve, será apresentado os benefícios das ontologias para os ACs.

No caso do sistema OntBot, os autores destacam a importância da ontologia para interpretação de sinônimos na base de conhecimento dos ACs e a atuação em diferentes domínios, permitindo responder perguntas complexas [Al-Zubaide and Issa 2011]. Em M-Learning, as ontologias contribuem com a base de conhecimento do AC, permitindo responder aos questionamentos realizados pelos usuários em vários idiomas, desde que haja tradutores [Abdelkefi and Kallel 2016].

[Augello et al. 2011] mostram a importância das ontologias na construção de um AC para um domínio específico, utilizando a ontologia Cyc e uma combinação com métodos probabilísticos. No exemplo construído, um AC auxilia estudantes na escolha de professores que serão orientadores em pesquisas. Já VOX System, com a ontologia, é capaz de interagir com o usuário realizando pesquisas inteligentes e retornando apenas resultados relevantes para um domínio de conhecimento específico. Além disso, o VOX System consegue lidar com diferentes domínios de pesquisa/conhecimento de forma multilíngue.

O uso de ontologia, no projeto TempLang, contribui para um modelo de linguagem capaz de responder a perguntas que exigem definições ou explicações e não se limita a um único domínio. A ontologia apoia o processo de raciocínio na resolução da questão, uma vez que a ontologia suporta o processo de inferência. Os trabalhos explicitados por [Ong and Soriano 2014] e [Macías-Galindo et al. 2011] reforçam a importância da ontologia na construção da base de conhecimento para que seja possível gerar um diálogo.

A interoperabilidade apresentada no sistema IMACA ocorre por meio do uso de ontologias, uma vez que estas permitem um compartilhamento de entendimento comum sobre as estruturas de informações entre diferentes subsistemas. A efetividade ocorrida nos sistemas apresentados por [Ong and Soriano 2014] e também existente no sistema IMACA só ocorre graças ao uso de ontologias que envolvem conhecimento acerca da emoção.

QP4: Quais as dificuldades na adoção de ontologias em chatbots no contexto de ensino-aprendizagem? 
Ao mesmo tempo que as ontologias auxiliam na construção da base de conhecimento, em uma visão geral, os autores discutem a dificuldade na limitação desta base de conhecimento. Conforme apresentado por [Al-Zubaide and Issa 2011], com o uso de ontologias há uma dificuldade em adicionar novos conhecimentos ao chatbot. $\mathrm{O}$ mesmo problema é identificado no trabalho de [Augello et al. 2011], que trata o uso de ontologias como um paradigma simbólico. Complementando, as ontologias não são suficientes para responder a perguntas que exigem explicações, como questões de comparação, factual e raciocínio [Freitas et al. 2014] apud [Dobrila 2009].

Com relação à construção da ontologia, o tempo gasto e a dificuldade são vistos como problemas. Em [Morrison and Rus 2013] é exposto que a construção manual de Ontologia gera excesso de trabalho e a construção semiautomática por corpos de texto recebe influências de pré-conceitos dos supervisores humanos, o que implica na necessidade de pesquisas científicas sobre o assunto. O VOX System identificou dificuldades de compreensão de significados da Wikipédia, pois apesar dos dados serem bastante estruturados, seu significado não é claro para o computador porquê não é representado em uma máquina processável (formalmente). Por último, a pesquisa de [Macías-Galindo et al. 2011] apresentou dificuldades para extrair dados de um domínio para gerar a base de conhecimento.

\section{QP: Como as ontologias podem contribuir para chatbots no contexto de ensino-aprendizagem?}

A pesquisa apresentada visa verificar a contribuição das ontologias para os ACs no contexto de ensino-aprendizagem. Ao fazer uma análise dos trabalhos resultantes foi possível levantar requisitos funcionais e não funcionais de um AC no contexto de ensinoaprendizagem, além disso, verificar os benefícios e problemas no uso de ontologias.

A contribuição das ontologias é visível uma vez que a QP3 (apresenta os benefícios das ontologias) coincide com o que é apresentado nas QP1 e QP2, que representam respectivamente os requisitos funcionais e não funcionais. De forma geral, um AC deve prover um diálogo em linguagem natural com um usuário. Para que isto ocorra, é necessário que tenha uma base de conhecimento consistente. De acordo com os trabalhos apresentados, esta é a principal contribuição das ontologias na construção de ACs.

Ao focar no contexto de ensino-aprendizagem, espera-se que este AC seja afetivo, possua uma boa usabilidade e interaja de diferentes maneiras. A afetividade nos trabalhos apresentados só foi possível por meio do uso de ontologias, assim como a possibilidade de interagir de diferentes maneiras, pois os subsistemas só conseguiram realizar comunicação com o uso de ontologias, que é um modelo formal e permite essa interação entre diferentes sistemas.

Este trabalho abre espaço para novas perspectivas, como por exemplo a inserção da metacognição em AC, a integração com aplicativos populares de mensagens, como apresentado por [Moreno et al. 2015] ao integrarem AC com o WhatsApp, e a aplicação do sistema em diferentes comunidades de estudantes. No entanto, a verificação da eficácia educacional dessas ferramentas é bastante complexa e desafiadora [Francisco et al. 2016], pois tecnologias não são neutras [Teixeira and Cukierman 2008] e, cada contexto cultural de aplicação pode gerar resultados extremamente diferentes, reforçando a necessidade de trabalhar com redes sociotécnicas para entender melhor os fenômenos. 
VI Congresso Brasileiro de Informática na Educação (CBIE 2017)

Anais do XXVIII Simpósio Brasileiro de Informática na Educação (SBIE 2017)

\section{Discussões finais}

A partir dos artigos científicos analisados, percebe-se que há uma dificuldade técnica em manter Ontologias atualizadas com a base de conhecimento necessária para os ACs atuarem em domínios diversos, pois o usuário do AC possui uma cultura onde a conversa precisa fazer sentido. O domínio de aplicação na Educação depende da criatividade e do diálogo, e observa-se que os ACs não conseguem dialogar com criatividade, e por isso estes devem ser ferramentas mediadoras quando se trata de educação. Projetos de AC para a educação bem sucedidos devem ter um escopo bem definido de diálogo.

Verifica-se a possibilidade, a partir de AC com Ontologias, de construir ambientes educacionais inovadores. Os trabalhos analisados apresentam ACs para auxiliar em resolução de atividades, aumentar a motivação, tirar dúvidas acerca de um assunto específico, dentre outros. Tal ferramenta se mostrou útil nos trabalhos analisados com relação ao contexto de ensino-aprendizagem, o que motiva a investigar ainda mais acerca do desenvolvimento e limites de atuação dos mesmos.

Espera-se, como trabalhos futuros, a investigação dos ACs como apoio em atividades colaborativas, e, junto aos resultados desta pesquisa, o desenvolvimento de um AC que seja capaz de aumentar a interação de estudantes em um ambiente colaborativo por meio de diálogos.

\section{Agradecimentos}

Os autores agradecem CNPq, FAPEMIG, CAPES, Facom/UFU, PROPP/UFU, IF Goiano e Unitri pelo apoio.

\section{Referências}

Abdelkefi, M. and Kallel, I. (2016). Conversational agent for mobile-learning: A review and a proposal of a multilanguage text-to-speech agent, "mobispeech". In Research Challenges in Information Science (RCIS), pages 1-6.

Al-Zubaide, H. and Issa, A. A. (2011). Ontbot: Ontology based chatbot. In Innovation in Information \& Communication Technology (ISIICT), 2011 Fourth International Symposium on, pages 7-12. IEEE.

Augello, A., Scriminaci, M., Gaglio, S., and Pilato, G. (2011). A modular framework for versatile conversational agent building. In Complex, Intelligent and Software Intensive Systems (CISIS), 2011 International Conference on, pages 577-582. IEEE.

Dobrila, T.-A. (2009). From semantic web knowledge to a functional conversational agent: A practical approach.

Fabian, R., Crăciunean, V., and Popa, E. M. (2006). Intelligent system modeling with total fuzzy grammars. In Proceedings of the 8th WSEAS international conference on Mathematical methods and computational techniques in electrical engineering, pages 82-87. World Scientific and Engineering Academy and Society (WSEAS).

Fonte, F. A. M., Burguillo, J. C., and Nistal, M. L. (2012). An intelligent tutoring module controlled by bdi agents for an e-learning platform. Expert Systems with Applications, 39(8):7546-7554. 
VI Congresso Brasileiro de Informática na Educação (CBIE 2017)

Anais do XXVIII Simpósio Brasileiro de Informática na Educação (SBIE 2017)

Francisco, R., Júnior, C. P., and Ambrósio, A. P. (2016). Juiz online no ensino de programação introdutória - uma revisão sitemática da literautra. In SBIE - Brazilian Symposium on Computers in Education, volume 27, page 11.

Freitas, H., Cury, D., and de Menezes, C. S. (2014). From ontologies to question-answer pairs-a template language for automated and customizable transformations. In New Perspectives in Information Systems and Technologies, pages 61-70. Springer.

Hussain, A., Cambria, E., Mazzocco, T., Grassi, M., Wang, Q.-F., and Durrani, T. (2012). Towards imaca: Intelligent multimodal affective conversational agent. In International Conference on Neural Information Processing, pages 656-663. Springer.

Kitchenham, B. (2004). Procedures for performing systematic reviews. Keele, UK, Keele University, 33(2004):1-26.

Lundqvist, K. O., Pursey, G., and Williams, S. (2013). Design and implementation of conversational agents for harvesting feedback in elearning systems. In European Conference on Technology Enhanced Learning, pages 617-618. Springer.

Macías-Galindo, D., Wong, W., Cavedon, L., and Thangarajah, J. (2011). Using a lexical dictionary and a folksonomy to automatically construct domain ontologies. In Australasian Joint Conference on Artificial Intelligence, pages 638-647. Springer.

Moraes, S. and Machado, R. (2016). Chatterbot for education: a study based on formal concept analysis for instructional material recommendation. In Brazilian Symposium on Computers in Education (Simpósio Brasileiro de Informática na Educação-SBIE), volume 27, page 1347.

Moreno, F., Manfio, E., Barbosa, C. R., and Brancher, J. D. (2015). Tical: Chatbot sobre o atlas linguístico do brasil no whatsapp. In Brazilian Symposium on Computers in Education (Simpósio Brasileiro de Informática na Educação-SBIE), volume 26, page 279.

Morrison, D. M. and Rus, V. (2013). The scholar legacy: A new look at the affordances of semantic networks for conversational agents in intelligent tutoring systems. In AIED Workshops.

Ong, E. C. J. and Soriano, Z. C. (2014). A conversational agent to shift students' affect state. In International Workshop on Empathic Computing, pages 86-97. Springer.

Ortony, A., Clore, G. L., and Collins, A. (1990). The cognitive structure of emotions. Cambridge university press.

Palangana, I. C. (2015). Desenvolvimento e aprendizagem em Piaget e Vigotski: a relevância do social. Summus Editorial.

Serban, I. V., Sordoni, A., Bengio, Y., Courville, A., and Pineau, J. (2016). Building end-to-end dialogue systems using generative hierarchical neural network models. In Thirtieth AAAI Conference on Artificial Intelligence.

Serón, F. J. and Bobed, C. (2016). Vox system: a semantic embodied conversational agent exploiting linked data. Multimedia Tools and Applications, 75(1):381-404.

Teixeira, C. A. N. and Cukierman, H. L. (2008). Modernidade, universalismo e assimetrias. Scientia, 19(2):94-101. 